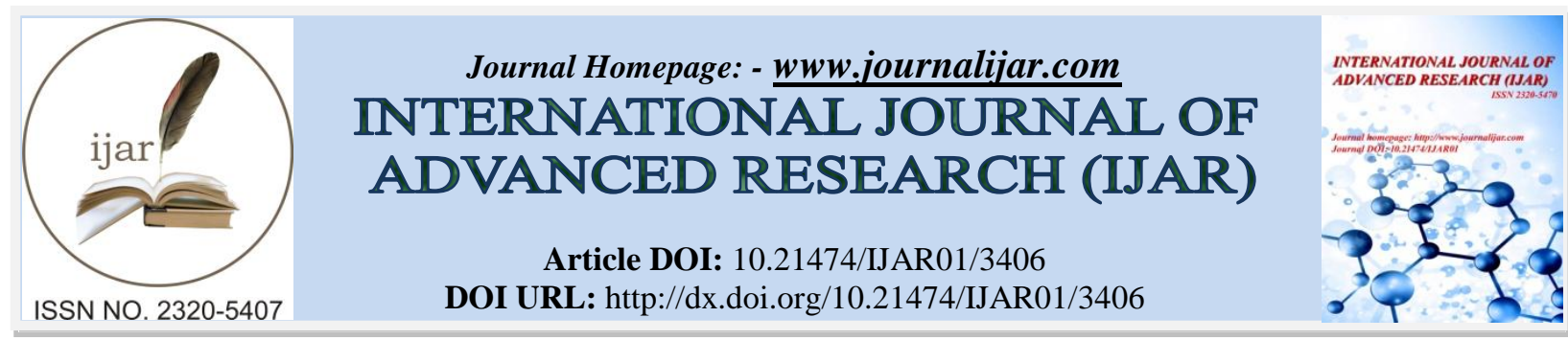

RESEARCH ARTICLE

\title{
TIME COURSE OF ALLODYNIA IN THE CHRONIC CONSTRICTION INJURY MODEL OF NEUROPATHIC PAIN.
}

\author{
Asma Alsaeed MBBS, Albatoul Almulhim MBBS, Fatimah Almajed MBBS, Fatimah Majrabi MBBS and \\ Karima Kahlat Asst prof. \\ king Faisal university, College of medicine.
}

\section{Manuscript Info}

Manuscript History

Received: 19 December 2016

Final Accepted: 14 January 2017

Published: February 2017

\begin{abstract}
Injury to the peripheral nerves results in chronic neuropathic pain that its mechanism is not well understood. Associated with neuropathic pain is allodynia, painful sensation to non-painful stimuli. Since the mechanism is not fully understood, there is yet no effective treatment for allodynia. The aim of this study is to monitor the time course of tactile allodynia after induced injury to the sciatic nerve. To achieve this aim, 4 Lewis rats were used. Baseline measurements were taken one day before performing chronic constrictive injury (CCI) model of neuropathic pain. Comparing with the contralateral paw, on the first day after CCI, the paw withdrawal threshold of the ipsilateral paw started to decrease, but did not reach the level of significance. The sixth day though, paw withdrawal threshold in ipsilateral was significantly different. Thus, successfully establishing allodynia. On the fifteenth day, measurements of paw withdrawal threshold increased, showing tendency to recovery from CCI. These changes may be related to different immune reactions to nerve injury. Knowing the role of different immune cells and relating them to the time course of allodynia development may give us an idea about the mechanism, and thus, an effective treatment.
\end{abstract}

Copy Right, IJAR, 2017,. All rights reserved.

\section{Introduction:-}

Pain is an undesirable sensation to intense stimuli. Depending on the intensity and duration of pain, it can be classified as either acute or chronic. There are many types of pain; each type is a clue of an underlying condition. Nociceptive pain is a type of protective pain to either external stimuli such as needle prick, or internal stimuli such as angina pain. Nociceptive pain stops whenever the noxious stimuli are handled and removed (1). Inflammatory pain which is the result of a tissue injury. In the inflamed area, sensations are exaggerated and prolonged to prevent further damage and to complete the healing process $(1 ; 2)$. Neuropathic pain results from nervous system damage. It is divided into two categories based on the location of injury: Central neuropathic pain, which can result from strokes or spinal cord injuries (3). The second type is peripheral nervous system injury, which we will focus on in our study. 
The peripheral nervous system responds to injury in two ways: axonal degeneration or segmental demyelination. Either ways, if the injury is too proximal and the cell body is to be affected, it will result in neuropathic pain. Injury to the peripheral nerves is usually associated with allodynia, which is pain produced by non-painful stimuli.

Studies of neuropathic pain are done more often on animal models, which experience different types of allodynia, which is feeling pain from non-painful stimuli, or hyperalgesia, increase sensitivity to painful stimuli. There are many mechanical injuries to the peripheral nerves that can induce allodynia: Chronic Constriction Injury (CCI) when applied on a rat model resulted in cold and mechanical allodynia (4). Partial Sciatic nerve Ligation (PSL) also induced mechanical allodynia (5). A study on the sciatic nerve was to observe a steady state of allodynia and hyperalgesia after transecting different combinations of the three branches of the nerve. Large levels of mechanical allodynia resulted after transection of tibial and sural nerves (6).

Mechanisms of neuropathic pain are still not fully understood despite the research that has been done. In this study, changes in allodynia after nerve injury at different time points will be investigated, which will add to the general understanding of the concept of neuropathic pain.

\section{Methods:-}

\section{Animals and surgery:-}

Male Lewis rats (Charles River, UK) at 11-13 weeks of age were used. All experimental animals were housed in standard plastic cages designed to allow easy access to food and water ad libitum. All animals were kept in appropriate room temperature $\left(24 \pm 1^{\circ} \mathrm{C}\right)$. All experiments complied with King Faisal University and international ethical guidelines for conduct of research on animals. Also, efforts were made to minimize the numbers of animals and their suffering. The CCI of the sciatic nerve was performed on rats anesthetized using isoflurane. During the surgical procedure, the sciatic nerve was exposed at the level of the middle of the thigh and freed of adhering tissue. Four ligatures ( 4.0 chromic gut) were tied loosely around the nerve such that its diameter was slightly constricted with about $1 \mathrm{~mm}$ spacing. After this, the incision was closed in layers by suturing of the muscle tissue and by metal clips on the skin. The surgery was performed under aseptic conditions.

\section{Allodynia measurement:-}

Paw withdrawal threshold (PWT) allows us to assess tactile allodynia by using automated von Fray-type system, which is the mechanical planter test apparatus (Ugo Basile, Milan, Italy). Before testing the rats, they are given a week to habituation to the settings. The tests are held at mornings, and the environment is kept under control. Baseline standard measurements of PWT were taken on day -1 , which is a day before the procedure, 10 min were allowed for rats to acclimate to the surroundings. $15 \mathrm{~min}$ to habituate over a mesh floor while placed in Perspex enclosure (Ugo Basile). Then, a metal filament is forced against the rats' planters that goes from $0 \mathrm{~g}$ to $50 \mathrm{~g}$ over 15 seconds. The paw withdrawal threshold can then be recorded by observing paw reflexes. The test is done three times with 2 minutes interval between each test stimulus. This is to avoid causing hypersensitivity in the rats' planters.

\section{Experimental design:-}

4 rats were used to study tactile allodynia changes at different time points. At first, before testing the rats, they were given a week of habituation to the surrounded settings. Next, the baseline measurements were taken on day (-1), the day before the surgery. Then, the CCI of the sciatic nerve was performed on day (0). After that, on the days post CCI (1), (6), and (15) the measurements were taken on the ipsilateral and contralateral paws of each rat. See figure (1).

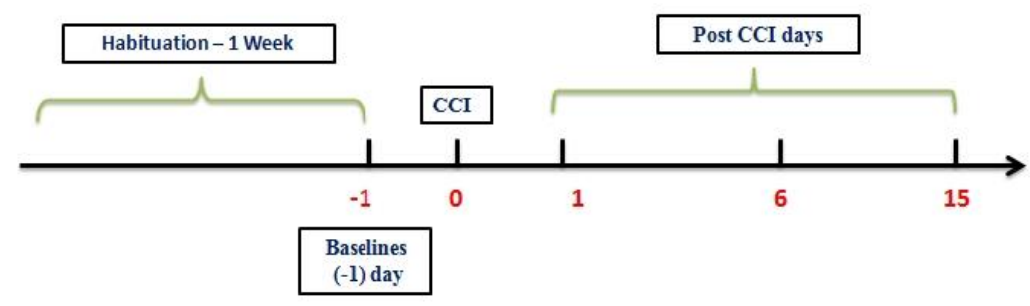

Figure 1:- Days of behavioral testing and CCI surgery. 


\section{Results:-}

General behavior of animals after CCI:-

In general, animals were looking well, drinking, eating, exploring and showing normal level of activities. After CCI, there were no reported illnesses or abnormalities in animals except that they were avoiding using the injured paw when stepping or putting their full weight on it. They were depending more on the non-injured paw when standing and walking.

\section{Time course of allodynia:-}

Figure 2 represents changes in paw withdrawal threshold measurement (g) at different time points by comparing the median of the paw withdrawal threshold for the contralateral and ipsilateral paw. On day -1 , which is the day for baseline measurements, there was no significant difference between the median of paw withdrawal threshold of the ipsilateral and the contralateral paw. Early after CCI on day 1, the median of the paw withdrawal threshold for the ipsilateral paw started to decrease, which indicates that the animals started to develop allodynia. On day 6 post-CCI, there was a significant decrease in the median of the ipsilateral paw withdrawal threshold compared to that of the contralateral paw $(\mathrm{p}<0.05)$. Indicating that allodynia was established in the injured paw. On day 15 post-CCI, the median paw withdrawal threshold in the ipsilateral paw showed a tendency to recovery from allodynia, as the median ipsilateral paw withdrawal threshold increased and had no statistically significant difference from that of the contralateral paw.

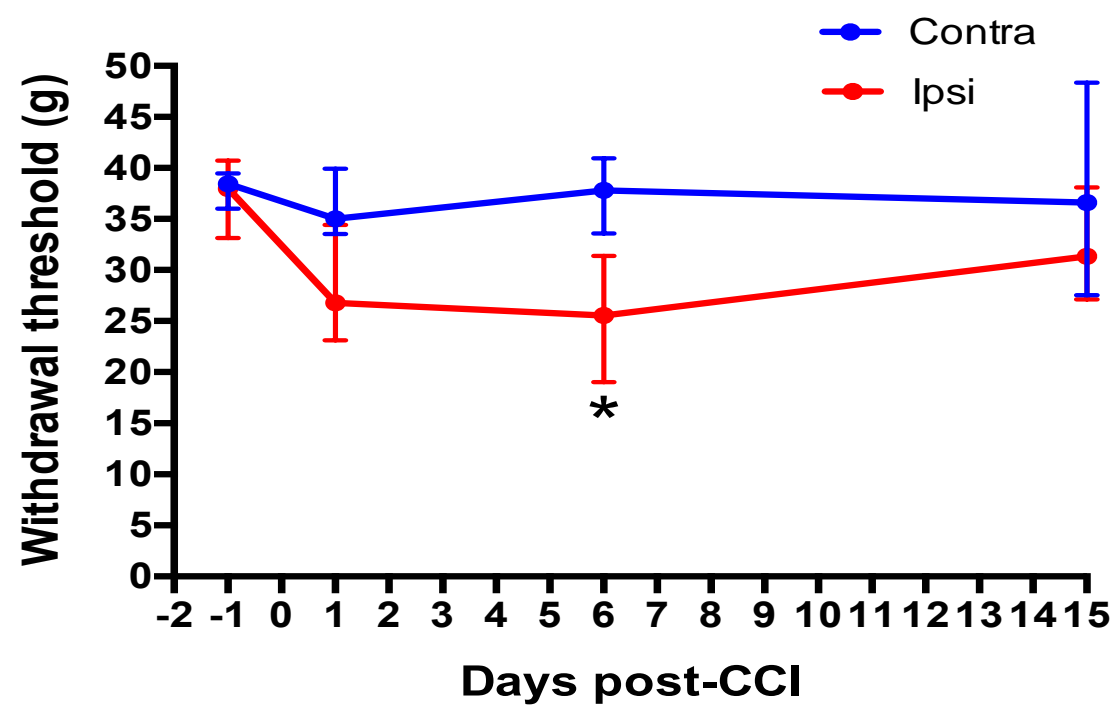

Figure 2:- Changes in paw withdrawal threshold measurements (g) at different time points. The blue line ( $\rightarrow$ Contra) refers to the contralateral paw. The red line $(\bullet-$ Insi) refers to the contralateral paw. The star $(*)$ indicates a significant difference $(\mathrm{p}<0.05)$ between the medians of the contralateral and the ipsilateral paw withdrawal threshold. Data are presented as medians and interquartile range.

Variability of allodynia measurements:-

Figure 3 shows that there is a behavioral variation between the animals' paw withdrawal thresholds ( $\mathrm{g}$ ) on different time points at both the contralateral and ipsilateral paws. This could be the result of genetic variation between the animals or due to technical difficulties. 

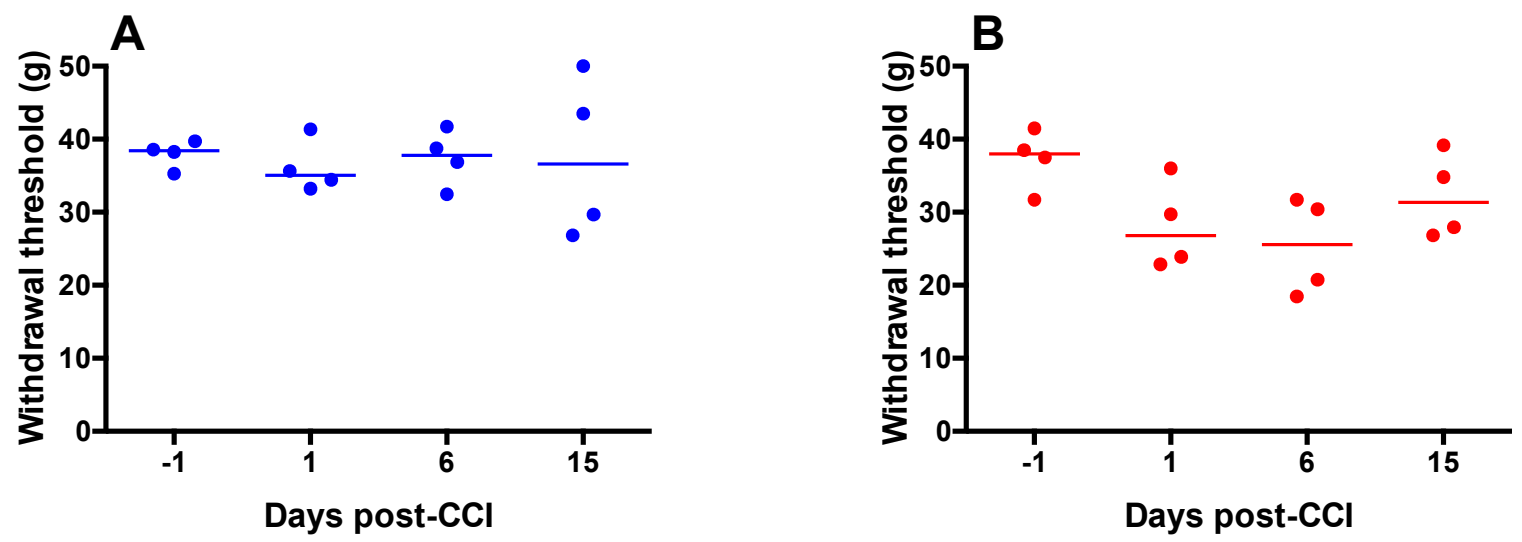

Figure 3:- Behavioral variability of animals in paw withdrawal thresholds at different time points. Graph (A) shows the behavioral variation between the animals in the contralateral paw. Graph $(\mathbf{B})$ shows the behavioral variation between the animals in the ipsilateral paw.

\section{Discussion:-}

Looking at the time course progression of allodynia after nerve injury may add to the understanding of the underlying mechanism of neuropathic pain. After exposing the rats, in the current study, to the chronic constriction injury (CCI) of the sciatic nerve, their paw withdrawal threshold to tactile stimuli has been measured. Compared to their contralateral side, their ipsilateral paw established allodynia with a significant difference 6 days after the procedure. Behavioral variations have been taken into consideration, and continuous assessment showed that on day 15 , the ipsilateral paw showed tendency to recovery where there was no significant difference with the contralateral paw.

Contribution of immune cells in nerve injury might have a direct effect on exaggerating neuropathic pain in the periphery (7). A study on partial nerve ligation (PNL) model had shown that at the site of injury, resident mast cells are the first respondent. Degranulation of mast cells due to activation releases proinflammatory mediators such as Histamine (8-10). These proinflammatory mediators have an algogenic effect on the site of damaged nerves (7). It was seen that giving histamine receptor antagonists to neuropathic rats suppresses mechanical allodynia, which means there is a direct connection between mast cells degranulation and allodynia (9). Thus, the mast cells may have contributed to the presence of allodynia in the first few days after the CCI (7).

During the early stages of neuropathic pain, neutrophils are recruited by mast cells to the injury area (11). In CCI model, it was observed that there was significant infiltration of neutrophils in the ipsilateral side of the rat. But there was no noticeable infiltration in the contralateral side (12). The contribution of neutrophils in neuropathic pain is that they are responsible for the release of proinflammatory mediators, such as chemokines that attract macrophages $(13 ; 14)$.

The phagocytic cells, macrophages, have a role in making neuropathic pain a chronic condition. Whenever there is an injury to the nerve, the blood-nerve barrier will allow circulating macrophages to join the resident macrophages in the nerve itself; this takes 2-3 days after injury (15). The hematogenously derived macrophages outnumber the resident macrophages and start releasing pronociceptive mediators $(7 ; 16)$. Along with the phagocytic function, this can explain the significant changes of allodynia on day 6. A study was done to test this concept by attenuating macrophages recruitment to the injured nerve. The result was reduction in neuropathic pain behaviors and delayed Wallerian degeneration of the nerve $(17 ; 18)$.

Resident mast cells and the recruited neutrophils and macrophages are not the only immune cells that were found in nerve injury area; T cells of both types were found in some nerve injury models (19). After CCI on athymic nude rats, which have no $T$ cells, mechanical allodynia was attenuated, which points to the role of $T$ cells in the establishment of allodynia (20). According to the cytokines produced by $\mathrm{T}$ cells, they can be divided into $\mathrm{T}$ helper type 1 and type 2 . In which, type 1 produce proinflammatory cytokines, and type 2 produce anti-inflammatory cytokines (7). Further investigations on the nude rats revealed that after transferring type 1 helper $\mathrm{T}$ cells, their 
levels of pain have increased. However, transferring type 2 cells gave an opposite result, it decreased their pain sensitivity (20). Thus, T helper Type 2 cells might have contributed to the tendency to recovery on day 15 .

Cytokines are important factors affecting neuropathic pain. Cytokines can be produced by the different immune cells, or one induces the production of others (7). Macrophages secrete many proinflammatory factors; one is IL- $1 \beta$ (21). The effect of IL-1 $\beta$ had been tested in the periphery by different administration roots. It resulted in prolonged allodynia and hyperagesia (22-25). When IL-1 $\beta$ binds to its receptors, it initiates several mechanisms resulting in inflammatory and nociceptor gene transcription (26;27). In CCI model, there is an up regulation in IL-1 $\beta$ mRNA $(28 ; 29)$. A study found that neutralizing antibodies to IL-1 $\beta$ receptor, IL-1 Type 1 receptor, caused reduction in associated pain behaviors in neuropathic rats $(30 ; 31)$. IL-1 $\beta$ has been considered as an algogenic agent that has a role in neuropathic pain (7).

In several neuropathic pain models, TNF- $\alpha$ mRNA are increased (32) along with increased protein expression (33;34), and enhanced expression of TNF- $\alpha$ receptors 1 and 2 after CCI (35) have been associated with development of allodynia.

Another cytokine, which is found to have a contribution in neuropathic pain mechanisms after CCI, is the proinflammatory cytokine IL-6 (19). It was seen that IL-6 knockout mice, genetically engineered mice that lack IL6, had an attenuated mechanical allodynia after CCI (36). Thus, there is a link between existence of IL-6 in injury site and mechanical allodynia (19).

Paw withdrawal thresholds were not the same in all rats that were tested. There was variability even when the rats were in the same circumstances. Such variance might be due to gene polymorphisms resulting in variation in pain sensitivity. Technical factors play an important role in this matter too. When placing the ligature around the sciatic nerve, we can never tie exactly in all rats. Thus, the amount of damage created is never the same.

\section{Conclusion:-}

Neuropathic pain is not the result of change in one cell; it is the interactions between immune cells and their proinflammatory secretions. Understanding the different actions of each, contribute to the general understanding of neuropathic pain. After analyzing the results, there was a significant difference in paw withdrawal threshold between ipsilateral and contralateral sides on the sixth day. Macrophages and Helper T cells Type 1 might be the cells contributing to this difference. Different cytokines secreted at the site of injury may have had a direct effect on the time-course of allodynia.

\section{References:-}

1. Michael C, Joachim S, Clifford JW. A maladaptive response of the nervous system to damage. Annual review of neuroscience. 2009;32:1 - 32 .

2. Juhl GI, Jensen TS, Norholt SE, Svensson P. Centrall sensitization phenomena after third molar surgery: a quantitative sensory testing study. Eur. J. Pain. 2008;12:116 - 27.

3. Ducreux D, Attal N, Parker F, Bouhassira D. Mechanism of central neuropathic pain: a combined psychophysical and fMRI study in syringomyelia. Brain. 2006;129:963 - 76.

4. Bennett $\mathrm{G}, \mathrm{Xie}$ Y. A peripheral mononeuropathy in rats that produces disorders of pain sensation like those in man. Pain. 1988;33:87 - 107.

5. Seltzer Z, Dubner R, Shir Y. A novel behavioral model of neuropathic pain disorders produced in rats by partial sciatic nerve injury. Pain. 1990;43:205 - 18.

6. Lee BH, Won R, Baik EJ, Lee SH, Moon CH. An animal model of neuropathic pain employing injury to the sciatic nerve branches. NeuroReport. 2000;11:657-61.

7. Michael AT, Anna KC, Fabien M, Stephen BM. Pathophysiology of neuropathic pain: Immune cells and molecules. International Anesthesia Research Society. 2007;105(3):838-47

8. Galli SJ, Nakae S, Tsai M. Mast cells in the development of adaptive immune responses. Nat Immunol 2005;6:135-42

9. Zue Y, Perkins NM, Tracey DJ, Geczy CL. Inflammation and hyperalgesia induced by nerve injury in the rat: a key role of mas cells. Pain 2003;105:467-79

10. Metcalfe DD, Baram D, Mekori YA. Mast cells. Physiol Rev 1997;77:1033-79 
11. Scapini P, Lapinet-Vera JA, Gasperini S, Calzetti F, Bazzoni F, Casatella MA. The neutrophil as a cellular source of chemokines. Immunol Rev 2000;177:195-203

12. Clatworthy AL, Illich PA, Castro GA, Walters ET. Role of peri-axonal inflammation in the development of thermal hyperalgesia and guarding behavior in a rat model of neuropathic pain. Neurosci Lett 1995;184:5-8

13. Witko-Sarsat V, Rieu P, Scamps-Latscha B, Lesavre P, Halbwachs Mecarelli L. Neutrophils: molecules, functions and pathophysiological aspects. Lab Invest 2000;80:617-53

14. Faurschou M, Borregaard N. Neutrophil granules and secretory vesicles in inflammation. Microbes Infect 2003;5:1317-27

15. Griffin JW, George R, Ho T. Macrophage systems in peripheral nerves. A review. J Neuropathol Exp Neurol 1993;52:553-60

16. Taskinen HS, Roytta M. The dynamics of macrophage recruitment after nerve transection. Acta Neuropathol (Berl) 1997; 93:252-9

17. Araki T, Sasaki Y, Milbrandt J. Increased nuclear NAD biosynthesis and SIRT1 activation prevent axonal degeneration. Science 2004;305:1010 -13

18. Ramer MS, French GD, Bisby MA. Wallerian degeneration is required for both neuropathic pain and sympathetic sprouting into the DRG. Pain 1997;72:71-8

19. Cui JG, Holmin S, Mathiesen T, Meyerson BA, Linderoth B. Possible role of inflammatory mediators in tactile hypersensitivity in rat models of mononeuropathy. Pain 2000;88:239-48

20. Moalem G, Xu K, Yu L. T lymphocytes play a role in neuropathic pain following peripheral nerve injury in rats. Neuroscience 2004;129:767-77

21. Giri JG, Lomedico PT, Mizel SB. Studies on the synthesis and secretion of interleukin 1. I. A 33,000 molecular weight precursor for interleukin 1. J Immunol 1985;134:343-9

22. Ferreira SH, Lorenzetti BB, Bristow AF, Poole S. Interleukin-1 beta as a potent hyperalgesic agent antagonized by a tripeptide analogue. Nature 1988;334:698-700

23. Follenfant RL, Nakamura-Craig M, Henderson B, Higgs GA. Inhibition by neuropeptides of interleukin-1 betainduced, prostaglandin-independent hyperalgesia. Br J Pharmacol 1989; 98:41-3

24. Watkins LR, Wiertelak EP, Goehler LE, Smith KP, Martin D, Maier SF. Characterization of cytokine-induced hyperalgesia. Brain Res 1994;654:15-26

25. Zelenka M, Schafers M, Sommer C. Intraneural injection of interleukin-1beta and tumor necrosis factor-alpha into rat sciatic nerve at physiological doses induces signs of neuropathic pain. Pain 2005;116:257-63

26. Pahl HL. Activators and target genes of Rel/NF-kappaB transcription factors. Oncogene 1999;18:6853-66

27. Tegeder I, Niederberger E, Schmidt R, Kunz S, Guhring H, Ritzeler O, Michaelis M, Geisslinger G. Specific inhibition of $\mathrm{I}\{$ kappa\}B kinase reduces hyperalgesia in inflammatory and neuropathic pain models in rats. $\mathrm{J}$ Neurosci 2004;24:1637-45

28. Okamoto K, Martin DP, Schmelzer JD, Mitsui Y, Low PA. Pro- and anti-inflammatory cytokine gene expression in rat sciatic nerve chronic constriction injury model of neuropathic pain. Exp Neurol 2001;169:386 $-91$

29. Kleinschnitz C, Brinkhoff J, Zelenka M, Sommer C, Stoll G. The extent of cytokine induction in peripheral nerve lesions depends on the mode of injury and NMDA receptor signaling. J Neuroimmunol 2004;149:77-83

30. Sommer C, Petrausch S, Lindenlaub T, Toyka KV. Neutralizing antibodies to interleukin 1-receptor reduce pain associated behavior in mice with experimental neuropathy. Neurosci Lett 1999;270:25-8

31. Schafers M, Brinkhoff J, Neukirchen S, Marziniak M, Sommer C. Combined epineurial therapy with neutralizing antibodies to tumor necrosis factor-alpha and interleukin-1 receptor has an additive effect in reducing neuropathic pain in mice. Neurosci Lett 2001;310:113-16

32. Wagner R, Myers RR. Schwann cells produce tumor necrosis factor alpha: expression in injured and noninjured nerves. Neuroscience 1996;73:625-9

33. Sommer C, Schafers M. Painful mononeuropathy in C57BL/Wld mice with delayed Wallerian degeneration: differential effects of cytokine production and nerve regeneration on thermal and mechanical hypersensitivity. Brain Res 1998;784: 154-62

34. George A, Schmidt C, Weishaupt A, Toyka KV, Sommer C. Serial determination of tumor necrosis factor-alpha content in rat sciatic nerve after chronic constriction injury. Exp Neurol 1999;160:124 -32

35. George A, Buehl A, Sommer C. Tumor necrosis factor receptor 1 and 2 proteins are differentially regulated during Wallerian degeneration of mouse sciatic nerve. Exp Neurol 2005;192: 163-6

36. Murphy PG, Ramer MS, Borthwick L, Gauldie J, Richardson PM, Bisby MA. Endogenous interleukin-6 contributes to hy- persensitivity to cutaneous stimuli and changes in neuropep- tides associated with chronic nerve constriction in mice. Eur J Neurosci 1999;11:2243-53 